Mykologie

\title{
Superaufgelöste Mikroskopie: Pilze unter Beobachtung
}

\begin{abstract}
SEBASTIAN SPUTH ${ }^{1}$, SABINE PANZER ${ }^{1}$, CHRISTIAN STIGLOHER ${ }^{2}$, ULRICH TERPITZ ${ }^{1}$ ${ }^{1}$ LEHRSTUHL FÜR BIOTECHNOLOGIE UND BIOPHYSIK, BIOZENTRUM, UNIVERSITÄT WÜRZBURG
\end{abstract}

2ZZENTRALE ABTEILUNG FÜR MIKROSKOPIE, BIOZENTRUM, UNIVERSITÄT WÜRZBURG

\section{The diffraction limit of light confines fluorescence imaging of sub-} cellular structures in fungi. Different super-resolution methods are available for the analysis of fungi that we briefly discuss. We exploit the filamentous fungus Fusarium fujikuroi expressing a YFP-labeled membrane protein showing the benefit of correlative light- and electron microscopy (CLEM), that combines structured illumination microscopy (SIM) and scanning election microscopy (SEM).

DOI: $10.1007 / \mathrm{s} 12268-021-1592-6$

(c) Die Autoren 2021

Um zellbiologische Vorgänge in Pilzen zu untersuchen, kennzeichnen Mykologen

bestimmte subzelluläre Strukturen mit spezifischen Fluoreszenzfarbstoffen oder fluo-

\begin{tabular}{|c|}
\hline A \\
\hline Ablauf von CLEM \\
Keimung der Sporen \\
Hochdruckgefrierung \\
Gefriersubstitution \\
Einbettung in Harz (LR-White) \\
Dünnschnitt (100 nm) \\
Antikörper / Zellwandfärbung \\
Fluoreszenzmikroskopie (SIM) \\
Kontrastierung / Kohlebeschichtung \\
REM-Aufnahmen \\
Korrelation der Bilddaten \\
3D-Modellierung
\end{tabular}
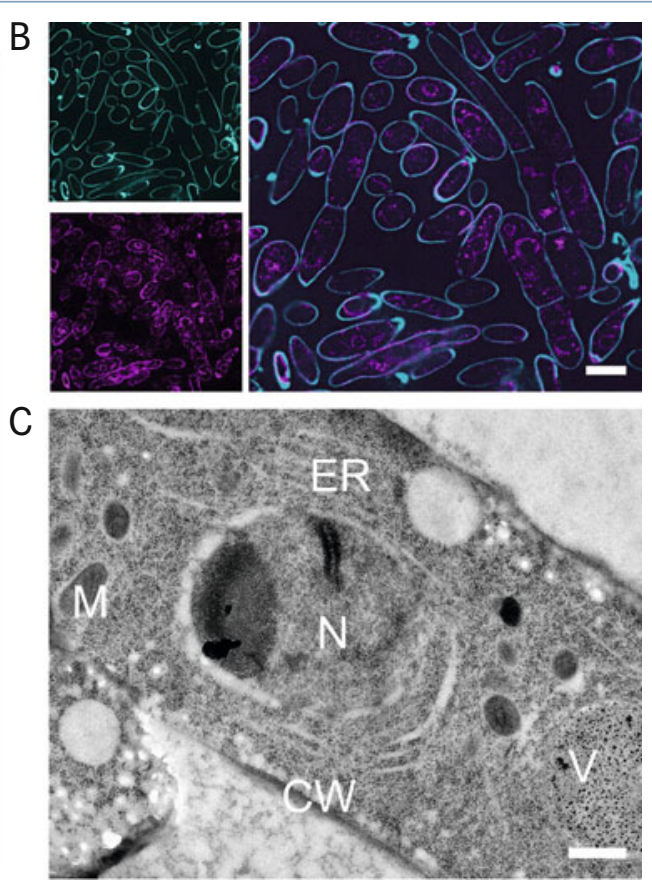

$\triangle$ Abb. 1: Korrelative Licht- und Elektronenmikroskopie (LEM) an Dünnschnitten (65-100 nm dick) von Fusarium fujikuroi-CarO-YFP in LR-White. A, Übersicht über den experimentellen Ablauf für die korrelative Licht- und Elektronenmikroskopie. B, SIM-Aufnahmen. Die Zellwand wurde mit Calcofluor eingefärbt (türkis, links oben), YFP mit einem mit Alexa488-markierten Antikörper (pink, links unten). Überlagerung rechts. Maßstab: $5 \mu \mathrm{m}$. C, TEM-Aufnahme. Die Ultrastruktur der Hyphe ist deutlich zu erkennen. CW: Zellwand; ER: Endoplasmatisches Retikulum; M: Mitochondrium; N: Nucleus; V: Vakuole. Maßstab: $500 \mathrm{~nm}$. reszierenden Proteinen wie dem gelb fluoreszierenden Protein (yellow fluorescent protein, YFP). Die fädigen Pilzzellen (Hyphen) sind meist sehr dünn und weisen Durchmesser zwischen zwei und drei Mikrometer auf. Bei der mikroskopischen Untersuchung der Hyphen stoßen Mykologen daher schnell auf ein Hindernis: das Abbe-Limit.

\section{Die mikroskopische Auflösung von Pilzen ist begrenzt}

Der Physiker und Optiker Ernst Abbe stellte im 19. Jahrhundert fest, dass aufgrund der Welleneigenschaften des Lichts keine noch so optimierte Optik fähig ist, Lichtstrahlen beliebig fein zu bündeln und dass die gebündelten Bereiche mindestens die Größe in etwa der halben Wellenlänge des eingesetzten Lichts haben. Anders ausgedrückt, können feine Details im mikroskopischen Untersuchungsobjekt nicht mehr getrennt dargestellt werden, wenn sie näher beieinanderliegen als ungefähr die halbe Wellenlänge des Lichts. Blaues Licht weist mit um die 400 Nanometer die kürzesten Wellenlängen innerhalb des sichtbaren Lichts auf. Damit liegt das Abbe-Limit je nach verwendeter Optik im Bereich von 150-200 Nanometern.

\section{Neue mikroskopische Verfahren} erhöhen die Auflösung

In den letzten Jahren wurden viele verschiedene Methoden entwickelt, die das AbbeLimit umgehen und die Auflösung der Fluoreszenzmikroskope deutlich verbessern. Diese Methoden werden unter dem Begriff „Superaufgelöste Fluoreszenzmikroskopie“ zusammengefasst.

Besonders deutlich ist die Auflösungsverbesserung bei der Lokalisationsmikroskopie, die Techniken wie PALM (photoactivated localisation microscopy) und dSTORM (direct stochastic optical reconstruction microscopy) umfasst [1]. Die hierbei verwendeten Fluorophore geben normalerweise nach Anregung spontan innerhalb weniger Nanosekunden längerwelliges und energieärmeres Licht ab (Emission). Jedoch ist es mithilfe von sehr starken Lichtintensitäten und Sauerstoff- 

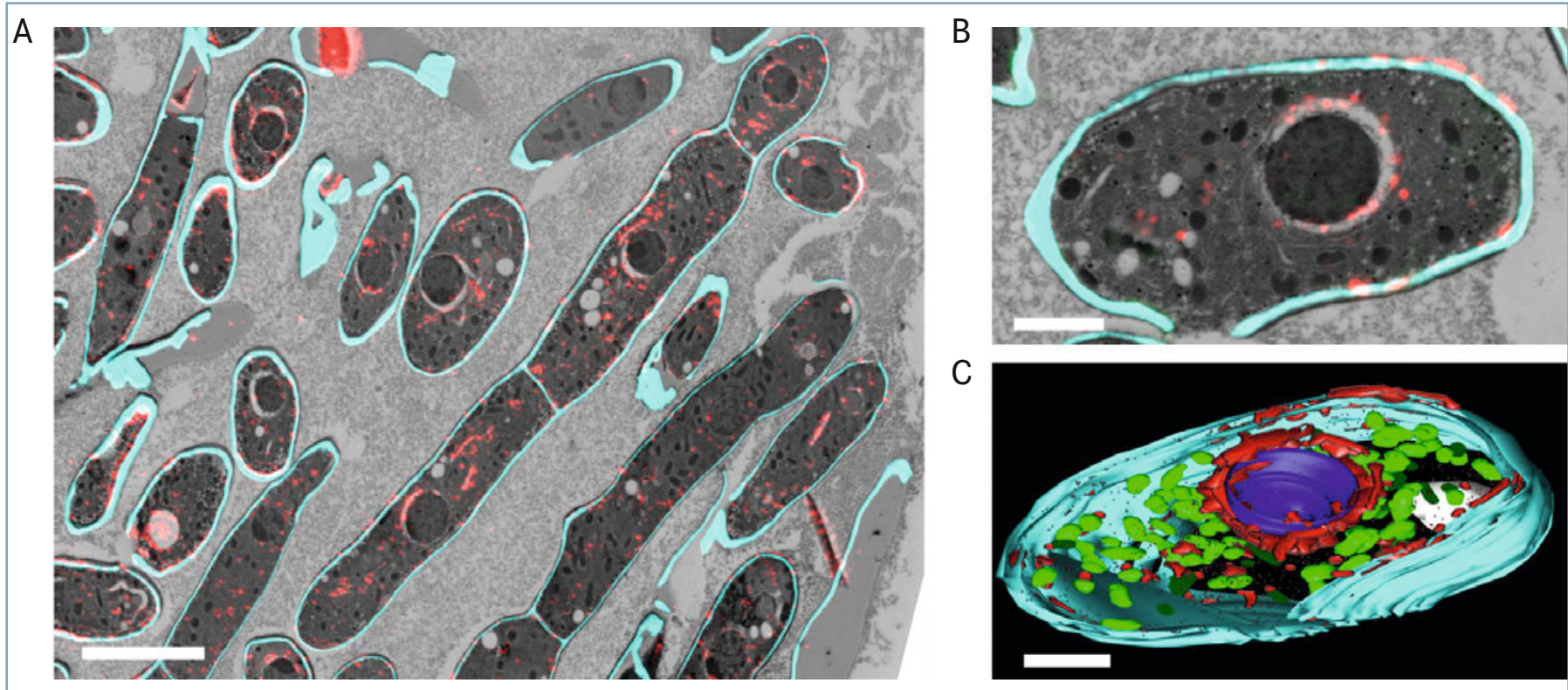

$\Delta$ Abb. 2: Korrelative Licht- und Elektronenmikroskopie von Fusarium fujikuroi-CarO-YFP. A, REM-Aufnahme mit korreliertem Fluoreszenzsignal der SIM-Aufnahmen mit Zellwand (türkis) und CarO-YFP (rot). Maßstab: 5 mm. B, Detailausschnitt. Von dieser Zelle wurde ein Z-Bildstapel erzeugt. Maßstab: $1 \mu \mathrm{m}$. C, Aus dem Bildstapel lässt sich nun ein Modell berechnen. Maßstab: $1 \mu \mathrm{m}$.

mangel möglich, die Fluorophore im dunklen Zustand zu stabilisieren. Betrachtet man eine solche Probe, blinken einzelne Fluorophore nur kurz auf, um anschließend lange Zeit dunkel zu bleiben. Mit sensitiven Hochgeschwindigkeitskameras kann dieses Blinken als Bilderserie aufgenommen werden. In jedem Bild erscheinen Fluorophore als Punktspreizfunktion mit einem Maximum, dessen Lage durch eine einfache mathematische Analyse bestimmt werden kann. Werden alle diese Maxima in ein neues Bild übertragen, entsteht ein rekonstruiertes neues Bild, das unter optimalen Bedingungen auch Strukturen auflösen kann, die weniger als 20 Nanometer Abstand haben. Lokalisationsmikroskopie erfordert allerdings insbesondere bei Pilzen teils intensive Protokolloptimierungen.

Seit wenigen Jahren gibt es für die Beobachtung von Strukturen jenseits des AbbeLimits einen neuen Ansatz, die Expansionsmikroskopie (ExM) [2]. Statt das Mikroskop zu modifizieren, vergrößert man bei dieser Technik die Probe selbst. Dies erreicht man, indem man Farbstoffe in einem Hydrogel verankert, das sich dann bei Entsalzung in Wasser ausdehnt. ExM wurde bereits erfolgreich an Pilzen wie Aspergillus fumigatus, Fusarium oxysporum und Ustilago maydis etabliert [3].

Für die regelmäßige Arbeit mit Pilzen hat sich in unserem Labor die Strukturierte Illuminationsmikroskopie (SIM) [4] bewährt. Bei
SIM befindet sich ein rotierendes Gitter im Strahlengang des Anregungslichts, das Beleuchtungsmuster in der Probe erzeugt. Das resultierende Fluoreszenzbild enthält auch Strukturinformationen jenseits der Auflösungsgrenze. Alle Bilder der verschiedenen Einstrahlwinkel werden miteinander verrechnet, und so wird ein neues superaufgelöstes Bild rekonstruiert. Die Aufnahmen können in verschiedenen Z-Ebenen erfolgen, um somit ein 3D-Bild zu erzeugen. SIM ist auch bei lebenden Zellen anwendbar. So lässt sich z. B. der pH-Gradient innerhalb von Pilzzellen darstellen, wie kürzlich am Maisbeulenbrand $U$. maydis gezeigt [5].

\section{Gemeinsame Fluoreszenz- und Elektronenmikroskopie}

Klassischerweise wird und wurde die Elektronenmikroskopie (EM) verwendet, um mit einer Auflösung von unter einem Nanometer Strukturinfomationen weit jenseits des AbbeLimits zu erzielen. Allerdings erfordert die EM im Vergleich zur Lichtmikroskopie langwierige Fixierungs- und Einbettungsschritte der Probe. Die Ausgabe erfolgt in Graustufen, Farben werden nicht dargestellt. Daher ist es bestechend, die Vorteile von EM und Fluoreszenzmikroskopie zu verbinden. Man nennt diese Technik korrelative Licht- und Elektronenmikroskopie (CLEM), wobei Fluoreszenzsignale in elektronenmikroskopischen Bildern einer Struktur zugeordnet werden können. Erst die verbesserte Auflösung der modernen fluoreszenzmikroskopischen Verfahren sowie die wachsende Rechenkapazität von Computern ermöglichte eine systematische und sinnvolle Verknüpfung der Bildinformationen von Fluoreszenz- und Elektronenmikroskopie.

Wir testeten, inwieweit dieser CLEMAnsatz an Pilzen praktiziert werden kann. Für diesen Versuch wählten wir den Schlauchpilz (Ascomycet) Fusarium fujikuroi, der das fluoreszenzmarkierte Membranprotein CarO-YFP, ein Rhodopsin, bildet [6]. Eine Übersicht des experimentellen Ablaufs zeigt Abbildung 1A; im Wesentlichen folgten wir dem Protokoll von Sebastian Markert et al. [7], mit einigen Anpassungen.

Die Pilzsporen (Konidien) keimen 15 Stunden und werden dann unter Hochdruck schockgefroren und somit im nahezu nativen Zustand kryoimmobilisiert. Anschließend erfolgt nach allmählicher Niedertemperaturentwässerung und Fixierung (Gefriersubstitution) mit Aceton und Ethanol als Lösungsmittel die Einbettung in das hydrophile Harz LR-White. In LR-White bleiben viele Proteinstrukturen erhalten, sodass spezifische Antikörper auch in den davon gefertigten Dünnschnitten binden können. Wir verwenden einen anti-YFP-Antikörper, der mit einem Fluoreszenzfarbstoff markiert ist und spezifisch das Membranprotein CarO-YFP erkennt. Außerdem färben wir die Zellwand mit Calcofluor, einem Farbstoff, der Chitin erkennt. Mit SIM ist im Dünnschnitt sowohl 
das anti-YFP-Signal als auch die Zellwand gut zu erkennen (Abb. 1B).

Im Anschluss an die Fluoreszenzmikroskopie erfolgt die Kontrastierung mit Uranylacetat und Bleicitrat sowie die Bedampfung mit einer sehr dünnen Kohleschicht zur Vorbereitung der Analyse am Rasterelektronenmikroskop (REM). Auch in den REM-Aufnahmen sind die Zellwände deutlich zu erkennen (Abb. 1C). Damit eignet sich die Zellwand als Referenzstruktur, um die beiden bildgebenden Verfahren miteinander zu korrelieren.

\section{Entwicklung eines Modells zur Darstellung des Pilzes}

Da die Ultradünnschnitte in Serie gefertigt werden, ist es möglich, Bildstapel zu erstellen, bei denen dieselbe Hyphe in unterschiedlichen Z-Ebenen zu sehen ist. Um SIMund REM-Daten zu korrelieren, müssen alle Bilder einer Schnittserie ausgerichtet, das Fluoreszenzsignal zugeordnet und schließlich über das EM-Bild gelegt werden (Abb. 2A). In unserem Beispiel ist deutlich erkennbar, dass das Rhodopsin CarO insbesondere im Endoplasmatischen Retikulum (ER) und in der Plasmamembran vorliegt. Mithilfe des Programms IMOD [8] kann ein 3D-Modell erzeugt werden (Abb. 2B, C), indem in jeder Ebene bestimmte Strukturen markiert werden, in unserem Beispiel das Rhodopsin, die Zellwand, der Zellkern und die Mitochondrien.

\section{Superaufgelöste Mikroskopie hilft bei der Erforschung von Pilzen}

In den letzten Jahren haben sich viele Möglichkeiten im Bereich der Mikroskopie ergeben. Für die Erforschung der Zellbiologie von Pilzen gibt es nunmehr eine Reihe von unterschiedlichen Methoden, um die Vorgänge in der Zelle jenseits des Abbe-Limits zu visualisieren. Die Korrelation von fluoreszenzmikroskopischen Daten mit der enormen Detailgenauigkeit von Hochdruck-Gefrier-EM-Präparaten ist eine vielversprechende Möglichkeit, um unser Verständnis von Pilzen voranzubringen.

\section{Danksagung}

Wir danken Nora Trinks und Jan Schlegel für die initialen CLEM-Experimente. Diese
Arbeit wurde gefördert durch die DFG (Projekt TE832/4-1).

\section{Literatur}

[1] Hohlbein J, Gryte K, Heilemann M, Kapanidis AN (2010) Surfing on a new wave of single-molecule fluorescence methods. Phys Biol 7: 031001

[2] Chen F, Tillberg PW, Boyden ES (2015) Optical imaging. Expansion microscopy. Science 347: 543-548

[3] Götz R, Panzer S, Trinks N et al. (2020) Expansion microscopy for cell biology analysis in fungi. Front Microbiol 11: 574

[4] Gustafsson MGL, Shao L, Carlton PM et al. (2008) Threedimensional resolution doubling in wide-field fluorescence microscopy by structured illumination. Biophys J 94: 49574970

[5] Panzer S, Brych A, Batschauer A, Terpitz U (2019) Opsin 1 and Opsin 2 of the corn smut fungus Ustilago maydis are green light-driven proton pumps. Front Microbiol 10: 735 [6] García-Martínez J, Brunk M, Avalos J, Terpitz U (2015) The CarO rhodopsin of the fungus Fusarium fujikuroi is a light-driven proton pump that retards spore germination. Sci Rep 5: 7798

[7] Markert SM, Bessereau J, Stigloher C et al. (2016) Filling the gap: adding super- resolution to array tomography for correlated ultrastructural and molecular identification of electrical synapses at the $C$. elegans connectome. Neurophotonics 3 : 41802
[8] Kremer JR, Mastronarde DN, McIntosh JR (1996) Computer visualization of three-dimensional image data using IMOD. J Struct Biol 116: 71-76

Funding note: Open Access funding enabled and organized by Projekt DEAL. Open Access: Dieser Artikel wird unter der Creative Commons Namensnennung 4.0 International Lizenz veröffentlicht, welche die Nutzung, Vervielfältigung, Bearbeitung, Verbreitung und Wiedergabe in jeglichem Medium und Forma erlaubt, sofern Sie den/die ursprünglichen Autor(en) und die Quelle ordnungsgemäß nennen, einen Link zur Creative Commons Lizenz beifügen und angeben, ob Änderungen vorgenommen wurden. Die in diesem Artikel genannten Creative Commons Lizenz, sofern sich aus der Abbildungslegende nichts anderes ergibt. Sofern das betreffende Material nicht unter der genannten Creative Commons Lizenz steht und die betreffende Handlung nicht nach gesetzlichen Vorschriften erlaubt ist, ist für die oben aufgeführten Weiterverwendungen des Materials die Einwilligung des jeweiligen Rechteinhabers einzuholen. Weitere Details zur Lizenz entnehmen Sie bitte der Lizenzinformation auf http://creativecommons.org/licenses/by/4.0/deed.de.

\section{Korrespondenzadresse:}

PD Dr. Ulrich Terpitz

Lehrstuhl für Biotechnologie und Biophysik

Universität Würzburg

Am Hubland

D-97074 Würzburg

ulrich.terpitz@uni-wuerzburg.de

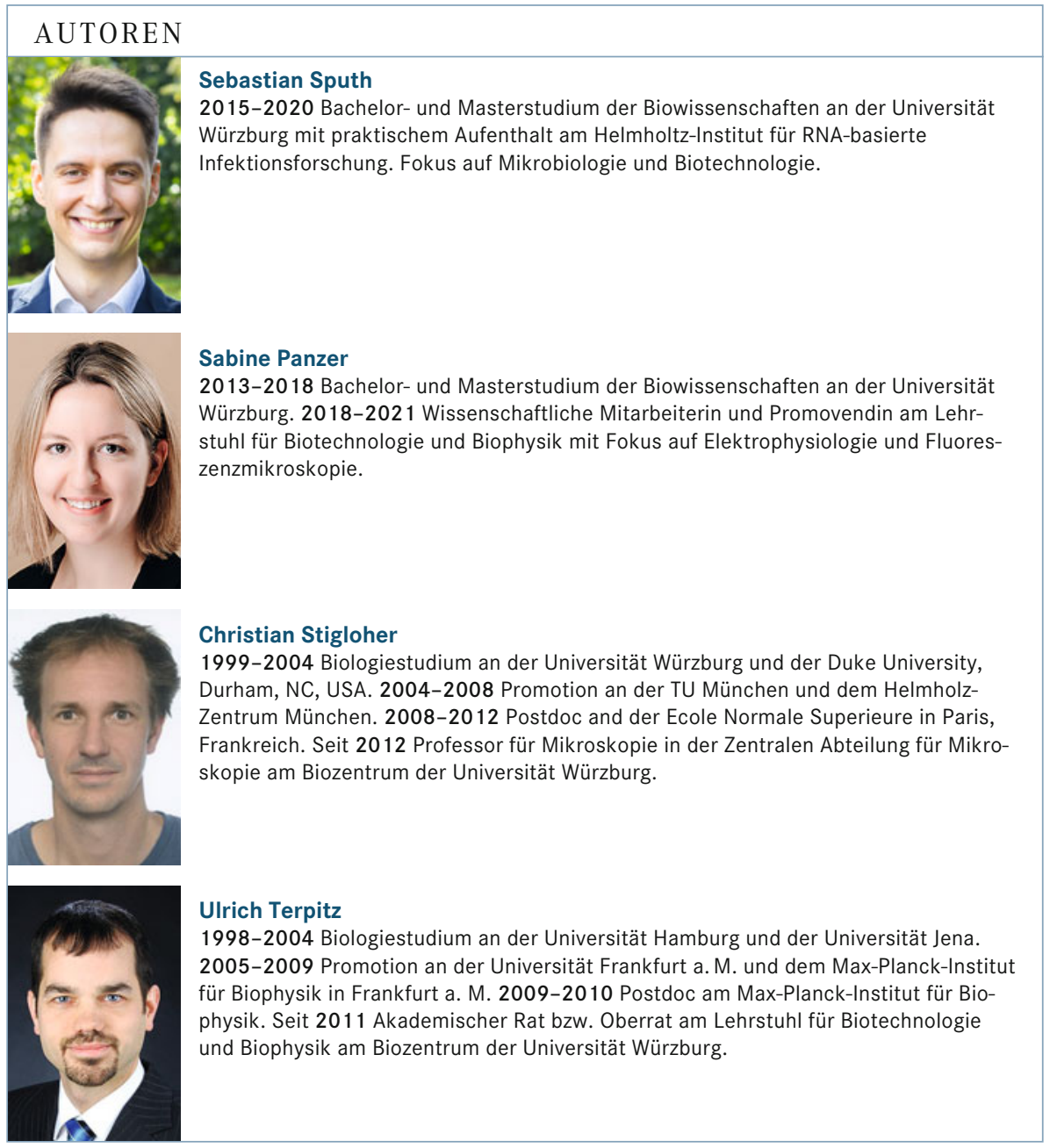

